\title{
Angiotensin Converting Enzyme Inhibitor and HMG-CoA Reductase Inhibitor as Adjunct Treatment for Persons with HIV Infection: A Feasibility Randomized Trial
}

\author{
Jason V. Baker ${ }^{1,2 *}$, Kathleen Huppler Hullsiek ${ }^{3}$, Rachel Prosser ${ }^{1}$, Daniel Duprez ${ }^{2}$, Richard Grimm ${ }^{1,2}$, \\ Russell P. Tracy ${ }^{4}$, Frank Rhame ${ }^{2,5}$, Keith Henry ${ }^{1,2}$, James D. Neaton ${ }^{3}$
}

1 Department of Medicine, Hennepin County Medical Center (HCMC), Minneapolis, Minnesota, United States of America, 2 Department of Medicine, University of Minnesota, Minneapolis, Minnesota, United States of America, 3 Department of Biostatistics, University of Minnesota, Minneapolis, Minnesota, United States of America, 4 Department of Biochemistry, University of Vermont, Burlington, Vermont, United States of America, 5 Department of Medicine, Abbott Northwestern Hospital, Minneapolis, Minnesota, United States of America

\begin{abstract}
Background: Treatments that reduce inflammation and cardiovascular disease (CVD) risk among individuals with HIV infection receiving effective antiretroviral therapy (ART) are needed.

Design and Methods: We conducted a $2 \times 2$ factorial feasibility study of lisinopril (L) (10 mg daily) vs L-placebo in combination with pravastatin (P) (20 mg daily) vs P-placebo among participants receiving ART with undetectable HIV RNA levels, a Framingham 10 year risk score $(F R S) \geq 3 \%$, and no indication for ACE-I or statin therapy. Tolerability and adherence were evaluated. Longitudinal mixed models assessed changes in blood pressure (BP), blood lipids, and inflammatory biomarkers from baseline through months 1 and 4.
\end{abstract}

Results: Thirty-seven participants were randomized and 34 [lisinopril/pravastatin $(n=9)$, lisinopril/P-placebo $(n=8), L-$ placebo/pravastatin $(n=9)$, L-placebo/P-placebo $(n=8)]$ attended at least one follow-up visit. Participants were $97 \%$ male, $41 \%$ white, $67 \%$ were current smokers, and $65 \%$ were taking a protease inhibitor. Median age was 48 years, CD4 count 483 cells $/ \mathrm{mm}^{3}$, FRS $7.79 \%$, total cholesterol $184 \mathrm{mg} / \mathrm{dL}$, and LDL-C $95 \mathrm{mg} / \mathrm{dL}$. There was no treatment difference for pravastatin vs P-placebo in total cholesterol, LDL-C, or any of the inflammatory biomarkers. Participants randomized to lisinopril vs. Lplacebo had significant declines in diastolic BP $(-3.3 \mathrm{mmHg}, \mathrm{p}=0.05)$, hsCRP $(-0.61 \mu \mathrm{g} / \mathrm{mL}, \mathrm{p}=0.02)$ and TNF- $\alpha(-0.17 \mathrm{pg} /$ $\mathrm{mL}, \mathrm{p}=0.04)$. Participants taking lisinopril vs $L$-placebo were more likely to report missed doses $(88$ vs $35 \% ; p=0.001)$ and have adherence $<90 \%$ by pill count ( 42 vs. $0 \% ; p=0.02)$. Few participants from either group reported side effects ( $n=3$ vs. $\mathrm{n}=1$ ).

Conclusions: The modest BP changes and decreased adherence with lisinopril and absence of lipid differences with pravastatin suggest future studies of these drug classes should consider a run-in period to assess adherence and use a different statin. Our results also indicate that ACE-I therapy may have anti-inflammatory benefits for ART-treated persons with HIV infection and this should be further evaluated.

Trial Registration: ClinicalTrials.gov NCT00982189

Citation: Baker JV, Huppler Hullsiek K, Prosser R, Duprez D, Grimm R, et al. (2012) Angiotensin Converting Enzyme Inhibitor and HMG-CoA Reductase Inhibitor as Adjunct Treatment for Persons with HIV Infection: A Feasibility Randomized Trial. PLoS ONE 7(10): e46894. doi:10.1371/journal.pone.0046894

Editor: Geneviève Chêne, Institut National de la Santé et de la Recherche Médicale, France

Received March 12, 2012; Accepted September 6, 2012; Published October 17, 2012

Copyright: (c) 2012 Baker et al. This is an open-access article distributed under the terms of the Creative Commons Attribution License, which permits unrestricted use, distribution, and reproduction in any medium, provided the original author and source are credited.

Funding: Funding for study provided by the American Heart Association Clinical Research Program (grant\# CRP2230357). More information on AHA funding can be found on their website: www.my.americanheart.org/professional/research/research_ucm_316889_subhomepage.jsp. The funders had no role in the study design, data collection and analysis, decision to publish, or preparation of the manuscript.

Competing Interests: The authors have declared that no competing interests exist.

*E-mail: baker459@umn.edu

\section{Introduction}

Individuals with HIV infection are at increased risk for premature cardiovascular disease (CVD) due to the higher prevalence of traditional risk factors (e.g., smoking), toxicity from antiretroviral therapy (ART; e.g., metabolic complications), as well as direct effects of HIV itself [1]. Specifically, HIV-related inflammation persists despite effective viral suppression with ART treatment and this may further amplify CVD risk
$[2,3,4,5]$. CVD prevention strategies that encompass both antiinflammatory benefits as well as traditional risk factor modification may be uniquely beneficial in this context.

Similar to the general population, high blood pressure (BP) and cholesterol account for a significant proportion of CVD risk among patients with HIV infection and remain a key component of prevention strategies [6]. In the general population, epidemiologic data demonstrate a consistent graded relationship between $\mathrm{BP}$ and cholesterol with GVD, which persists through normal BP 
values (down to at least $115 / 75 \mathrm{mmHg}$ ) and moderate total cholesterol levels (155 to $>200 \mathrm{mg} / \mathrm{dL}$ ) [7,8]. For a target population at higher absolute CVD risk, such as individuals with HIV infection, these data suggest risk factor reductions may be beneficial irrespective of whether individual BP or cholesterol levels exceed current thresholds for treatment $[9,10]$.

Angiotensin converting enzyme inhibitors (ACE-I) and HMGCoA reductase inhibitors ('statins') have been shown to reduce CVD risk through their $\mathrm{BP}$ and cholesterol lowering properties, respectively $[11,12]$. However, both classes of medications appear to have additional anti-inflammatory pleotropic effects that may be uniquely beneficial for HIV positive patients [13,14,15]. Prior to expanding the use of ACE-I and/or statins for HIV-infected persons to patients for whom these treatments are not currently indicated, safety and tolerability data are needed to inform largescale trials that more clearly define the net risk-benefit balance.

The goal of this study was to determine if a strategy using lisinopril (an ACE-I) at $10 \mathrm{mg}$ daily and pravastatin (a 'statin') at $20 \mathrm{mg}$ daily as adjunctive treatment was feasible, well tolerated, and led to risk factor reductions when given alone or in combination to virologically suppressed patients receiving ART. We also explored the potential treatment effect on biomarkers of systemic inflammation: high sensitivity C-reactive protein (hsGRP), interleukin-6 (IL-6) and tumor necrosis factor-alpha $(\mathrm{TNF}-\alpha)$.

\section{Methods}

The protocol for this trial and supporting CONSORT checklist are available as supporting information; see Checklist S1 and Protocol S1.

\section{Participants}

Participants with HIV infection receiving ART with HIV RNA levels $<200$ copies/mL and a FRS $\geq 3 \%$ for 10 -year coronary heart disease risk were enrolled after written informed consent at one of two HIV clinics (Hennepin County Medical Center [HCMC] and Clinic 42, Allina Hospitals and Clinics, Minneapolis, Minnesota) from January 2010 through February 2011. Exclusion criteria included known CVD, hypertension or BP $\geq 140 / 90 \mathrm{mmHg}$, low-density lipoprotein cholesterol (LDL-C) $>160 \mathrm{mg} / \mathrm{dL}$ (or $>130 \mathrm{mg} / \mathrm{dL}$ with a FRS $>10 \%$ ), triglycerides $>500 \mathrm{mg} / \mathrm{dL}$, diabetes, cirrhosis, serum creatinine $\geq 2.0 \mathrm{mg} / \mathrm{dL}$, or a contra-indication to taking ACE-I or statin therapy. Our criteria specifying a FRS $\geq 3 \%$ both eliminates those at very low risk for CVD and efficiently selects for a target population at moderate CVD risk but for whom BP or cholesterol lowering therapy were not typically indicated. When applying data that HIV infection is associated with approximately a 2-fold increased CVD risk, our target population should have at least a 5\% risk for coronary event in next 10 years [16]. FRS used for analyses was calculated from the published algorithm that considered age, gender, systolic BP, total cholesterol, high-density lipoprotein [HDL-G], and current smoking and diabetes status [17]. For screening purposes, FRS was estimated through application of a point-of-care online calculator (hp2010.nhlbihin.net/atpiii/calculator.asp). The study was approved by the institutional review board at each clinical site (HCMC Human Subjects Research Committee and Allina Hospitals and Clinics Institutional Review Board) and the protocol was registered at ClinicalTrials.gov (NCT00982189).

\section{Study Design}

The study design was a randomized, double-blinded $2 \times 2$ factorial design of lisinopril (L) $10 \mathrm{mg}$ daily versus matched Lplacebo daily in combination with pravastatin (P) $20 \mathrm{mg}$ daily versus P-placebo (Figure 1). The goal of the study was to inform planning for larger studies by first assessing ability to recruit for the proposed interventions, and assessing tolerability and adherence. Planned sample size was 40 participants, which provided $80 \%$ power to detect a $12.6 \mathrm{mmHg}$ difference between the lisinopril and L-placebo groups for systolic BP (assuming a standard deviation of $14 \mathrm{mmHG}$ ) and a $27 \mathrm{mg} / \mathrm{dL}$ difference in LDL cholesterol between the pravastatin and P-placebo groups (assuming a standard deviation of $30 \mathrm{mg} / \mathrm{dL}$ ). The sample size and recruitment period was limited by budget and time restrictions associated with the American Heart Association funding mechanism supporting the study.

In order to blind both participants and study investigators to the treatment assigned, active study drugs were over-encapsulated to match the respective placebo capsules. Treatment allocation was balanced in blocks of 4 or 8 providing four groups of approximately equal size. Treatment schedules were only known to the unblinded statistician and the pharmacist responsible for preparing study medication bottles; neither had contact with study participants. After the baseline assessment, participants were instructed to take 1 capsule from each study medication bottle by mouth daily and returned for repeat study visit procedures at 1 and 4 months.

\section{Outcomes}

Adherence was assessed via participant self-reported estimates of number of missed doses per week during the study, and then objectively at month 4 by pill count. Tolerability and safety were ascertained through participant history at both 1 and 4 month visits. A fasting lipid profile (total cholesterol, LDL-C, HDL-C, and triglycerides) was obtained at the site clinical laboratory at baseline, 1 and 4 months. Research nurses measured BP in triplicate at each study visit, with mean values used for analyses. Plasma biomarkers of systemic inflammation were also assessed at baseline and the 1 and 4-month follow-up visits. Inflammatory markers were measured by the Laboratory for Clinical Biochemistry Research at the University of Vermont; hsCRP was measured

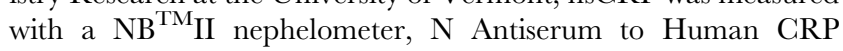
(Siemens Diagnostics, Norwood, MA); IL-6 with Chemiluminescent Sandwich ELISA (R\&D Systems, Minneapolis, MN); and TNF- $\alpha$ with Millipore Panel B multiplex (Billerica, MA). The lower level of detection for hsCRP, IL- 6 , and TNF- $\alpha$ were $0.16 \mu \mathrm{g} / \mathrm{mL}, 0.16 \mathrm{pg} / \mathrm{mL}$, and $0.32 \mathrm{pg} / \mathrm{mL}$. All samples were analyzed blinded to treatment group. The assay coefficient of variance $(\mathrm{CV})$ using these methods is $5 \%$ for hsCRP, $7 \%$ for IL-6, and $8 \%$ for TNF- $\alpha$. In addition to these measures we also obtained a basic metabolic panel, aspartate aminotransferase, alinine aminotransferase, serum creatine kinase, and a complete blood count, and HIV clinical labs (HIV RNA level and CD4+ T-cell count) at each visit.

\section{Statistical Methods}

All analyses are based on the participants who had at least one follow-up visit. Descriptive statistics were calculated to compare self-reported side-effects and medication adherence and pill counts for each treatment (lisonopril versus L-placebo and pravastatin versus P-placebo); $\mathrm{p}$-values for those comparisons are from Fisher's exact tests. A missed follow-up visit was assigned the worse adherence category ( $>3$ misses per week). Longitudinal mixed effects regression models which used all available follow-up data 
HIV-infected participants with undetectable HIV RNA, no indication for ACE-I or 'statin' use and a 10-year FRS $\geq 3 \%$

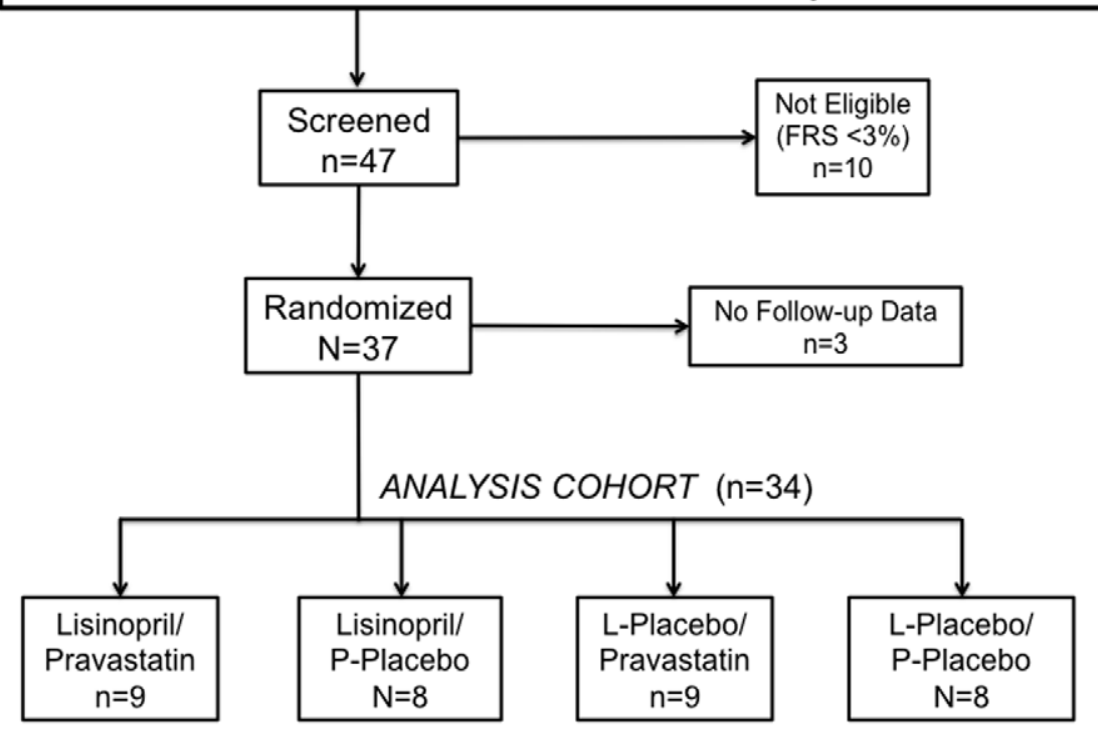

Figure 1. Study Design Flow-Diagram. doi:10.1371/journal.pone.0046894.g001

were used to assess treatment effects on change in BP, lipids, and inflammatory biomarkers averaged over both month 1 and 4 visits. Models included the baseline level of the outcome of interest, main effects for lisinopril and pravastatin, and the interaction between those treatments. None of the interaction terms were significant and therefore they were removed from the final models; only the results of main effects models are presented. Levels of hsCRP, IL-6 and TNF- $\alpha$ were $\log _{\mathrm{e}}$-transformed before analysis; results are reported on the original scale after back-transformation. A global assessment measure described by O'Brien was also considered for the inflammatory biomarkers since we expected the treatments to have a similar effect on all of markers [18]. With this "rank-sum" method the values of hsCRP, IL- 6 and TNF- $\alpha$ were ranked and then summed for each treatment group. Models as described above were used to compare the treatments for sum of the ranks. The comparisons of the inflammatory markers are considered exploratory. Two-sided p-values and 95\% confidence intervals (CIs) are cited, with no adjustments for multiple comparisons. Analyses were performed using SAS version 9.2 (SAS Institute) and $\mathrm{R}$ version 2.9 .

\section{Results}

\section{Study Population}

Forty-seven participants completed a screening visit and 37 of these were randomized (Figure 1). The reason for screening failure $(\mathrm{n}=10)$ was a FRS $<3 \%$. Three persons who were randomized withdrew consent prior to starting study medication and had no follow-up data (2 allocated to lisinopril/P-placebo group and 1 allocated to L-placebo/P-placebo group). Of the remaining 34 participants in the final analysis cohort, one missed the month 1 visit (from L-placebo/P-placebo group) and one missed the month 4 visit (from Lisinopril/P-placebo group). Baseline characteristics for the study population are presented in Table 1. Median age was 48, only one female was randomized, the majority $(68 \%)$ reported smoking cigarettes, median CD4 count was 482 cells $/ \mathrm{mm}^{3}$, and most $(65 \%)$ participants were taking protease-inhibitor-based ART. No participants had a prior history of injection drug use. Median FRS was 7.9\%, and BP and cholesterol levels were below treatment thresholds for persons without prior GVD. There were no significant differences in baseline characteristics between lisinopril and L-placebo groups, pravastatin and P-placebo groups, or when compared across all 4 of the individual treatment combinations.

\section{Tolerability and Adherence}

Few participants reported side effects to study medication during the 4-month study period (Table 2). One participant each in the lisinopril and L-placebo groups reported cough. The participant receiving active lisinopril was unblinded and stopped the study medication. Additional specific side effects reported include nausea $(\mathrm{n}=1)$ and runny nose $(\mathrm{n}=1)$. Despite no difference in self-reported side effects, participants taking lisinopril vs. L-placebo reported lower rates of perfect adherence (i.e., no missed doses) at month $1(59 \%$ vs. $100 \% ; \mathrm{p}=0.007)$ and month 4 $(13 \%$ vs. $65 \% ; p=0.007)$. Of the participants who returned study medication $(\mathrm{n}=25)$, adherence of $>90 \%$ of possible doses (assessed by pill count) was achieved by $58 \%$ of those randomized to lisinopril and $100 \%$ of those taking L-placebo $(p=0.01)$. Of participants who did not return study medications at month 4 , one discarded his remaining supply and the others failed to return the bottles after repeated requests by the study coordinator. Side effects and adherence (assessed by self report or via pill count) did not differ between pravastatin and P-placebo groups (Table 2).

\section{Treatment Effect of Lisinopril on Blood Pressure}

Compared to L-placebo, declines in systolic and diastolic BP were greater at both 1 and 4 months and averaged across both visits. For the latter, the treatment difference averaged across follow-up was $-2.6 \mathrm{mmHg}(95 \% \mathrm{CI}-8.1,2.8 ; \mathrm{p}=0.33)$ for systolic $\mathrm{BP}$ and $-3.3 \mathrm{mmHg}(95 \% \mathrm{CI}-6.5,-0.1 ; \mathrm{p}=0.05)$ for diastolic BP (Table 3 and Figure 2). 
Table 1. Baseline Characteristics.

\begin{tabular}{|c|c|c|c|c|c|}
\hline & Overall & Lisinopril & L-placebo & Pravastatin & P-placebo \\
\hline Totals (N) & 34 & 17 & 17 & 18 & 16 \\
\hline Age (years), median [IQR] & $48[44,56]$ & $49[45,53]$ & $47[44,56]$ & $48[45,56]$ & $51[44,55]$ \\
\hline Male gender (\%) & 33 (97\%) & 17 (100\%) & 16 (94\%) & 17 (94\%) & $16(100 \%)$ \\
\hline \multicolumn{6}{|l|}{ Race/ethnicity } \\
\hline White (\%) & $14(41 \%)$ & $5(29 \%)$ & $9(53 \%)$ & $7(39 \%)$ & 7 (44\%) \\
\hline African Am (\%) & $16(47 \%)$ & $11(65 \%)$ & $5(29 \%)$ & $9(50 \%)$ & $7(44 \%)$ \\
\hline Hispanic (\%) & $3(9 \%)$ & 0 & $3(18 \%)$ & $1(6 \%)$ & $2(13 \%)$ \\
\hline Other (\%) & $1(3 \%)$ & $1(6 \%)$ & 0 & $1(6 \%)$ & 0 \\
\hline IDU, never (\%) & $34(100 \%)$ & $17(100 \%)$ & $17(100 \%)$ & $18(100 \%)$ & $16(100 \%)$ \\
\hline Smoker (\%) & $23(68 \%)$ & $13(76 \%)$ & $10(59 \%)$ & $11(61 \%)$ & $12(75 \%)$ \\
\hline Hepatitis B or C (\%) & $11(33 \%)$ & $4(24 \%)$ & $7(41 \%)$ & $4(22 \%)$ & $7(44 \%)$ \\
\hline ART Regimen, PI-based (\%) & $22(65 \%)$ & $8(47 \%)$ & $14(82 \%)$ & $11(61 \%)$ & $11(69 \%)$ \\
\hline CD4 cells $/ \mathrm{mm}^{3}$,median [IQR] & $483[310,609]$ & $429[320,595]$ & $522[288,609]$ & $417[288,535]$ & $579[367,708]$ \\
\hline \multicolumn{6}{|l|}{ CVD RISK FACTORS } \\
\hline SBP $(\mathrm{mmHg})$, median [IQR] & $123[117,126]$ & $122[118,125]$ & $123[117,128]$ & $124[117,128]$ & $122[114,126]$ \\
\hline DBP $(\mathrm{mmHg})$, median [IQR] & $76[70,79]$ & $76[71,77]$ & $77[70,83]$ & $77[70,79]$ & $74[70,80]$ \\
\hline T. Chol (mg/dL), median [IQR] & $184[162,196]$ & $182[162,196]$ & $185[165,195]$ & $187[165,195]$ & $179[158,197]$ \\
\hline LDL-C (mg/dL), median [IQR] & $95[86,115]$ & $93[78,110]$ & $98[91,119]$ & $96[86,119]$ & $95[85,111]$ \\
\hline HDL-C (mg/dL), median [IQR] & $42[35,54]$ & $51[38,60]$ & $35[35,49]$ & $43[35,53]$ & $42[35,76]$ \\
\hline FRS 10-year, median [IQR] & $7.9[4.7,10.6]$ & $7.6[6.5,10.6]$ & $8.0[4.5,10.2]$ & $7.9[5.9,9.4]$ & $7.8[4.5,11.0]$ \\
\hline \multicolumn{6}{|l|}{ BIOMARKERS } \\
\hline hsCRP $(\mu \mathrm{g} / \mathrm{mL})$, median [IQR] & $\begin{array}{l}1.37 \\
{[0.55,2.52]}\end{array}$ & $\begin{array}{l}1.34 \\
{[0.56,2.25]}\end{array}$ & $\begin{array}{l}1.40 \\
{[0.55,2.52]}\end{array}$ & $\begin{array}{l}1.74 \\
{[0.56,2.93]}\end{array}$ & $\begin{array}{l}1.30 \\
{[0.44,1.97]}\end{array}$ \\
\hline IL-6 (pg/mL), median [IQR] & $\begin{array}{l}1.47 \\
{[0.95,2.31]}\end{array}$ & $\begin{array}{l}1.57 \\
{[0.95,2.82]}\end{array}$ & $\begin{array}{l}1.37 \\
{[0.95,2.17]}\end{array}$ & $\begin{array}{l}1.37 \\
{[1.2,2.31]}\end{array}$ & $\begin{array}{l}1.60 \\
{[0.90,2.49]}\end{array}$ \\
\hline TNF- $\alpha(\mathrm{pg} / \mathrm{mL})$, median [IQR] & $\begin{array}{l}3.99 \\
{[2.99,4.62]}\end{array}$ & $\begin{array}{l}4.51 \\
{[3.81,4.81]}\end{array}$ & $\begin{array}{l}3.72 \\
{[2.99,4.17]}\end{array}$ & $\begin{array}{l}4.13 \\
{[2.99,4.99]}\end{array}$ & $\begin{array}{l}3.85 \\
{[2.94,4.53]}\end{array}$ \\
\hline
\end{tabular}

doi:10.1371/journal.pone.0046894.t001

Table 2. Toxicity and Adherence During Follow-up.

\begin{tabular}{|c|c|c|c|}
\hline & Lisinopril & L-Placebo & p-value ${ }^{1}$ \\
\hline Side Effects (anytime during study) & $18 \%(3)$ & $6 \%(1)$ & 0.60 \\
\hline Cough & $6 \%(1)$ & $6 \%(1)$ & \\
\hline Missed Doses/Week (self-report at end of study) & & & 0.001 \\
\hline None & $12 \%(2)$ & $65 \%(11)$ & \\
\hline 1-2 misses/week & $24 \%(4)$ & $24 \%(4)$ & \\
\hline$\geq 3$ or declined to specify & $65 \%(11)$ & $12 \%(2)$ & \\
\hline \multirow{2}{*}{$\begin{array}{l}>90 \% \text { Adherence (Pill count at end of study; data available for } n=12 \text { in Lisinopril group and } n=13 \text { in } \\
\text { L-Placebo group) }\end{array}$} & $58 \%(7)$ & $100 \%(13)$ & 0.01 \\
\hline & Pravastatin & P-Placebo & p-value \\
\hline Side Effects (anytime during study) & $11 \%(2)$ & $13 \%(2)$ & $>0.99$ \\
\hline Missed Doses/Week (self-report at end of study) & & & $>0.99$ \\
\hline None & $39 \%(7)$ & $38 \%(6)$ & \\
\hline 1-2 misses/week & $22 \%(4)$ & $25 \%(4)$ & \\
\hline$\geq 3$ or declined to specify & $39 \%(7)$ & $38 \%(6)$ & \\
\hline $\begin{array}{l}>90 \% \text { Adherence (Pill count at end of study; data available for } n=15 \text { in Pravastatin group and } n=10 \text { in } \\
\text { P-Placebo group) }\end{array}$ & $80 \%(12)$ & $80 \%(8)$ & $>0.99$ \\
\hline
\end{tabular}




\section{A) Systolic Blood Pressure}

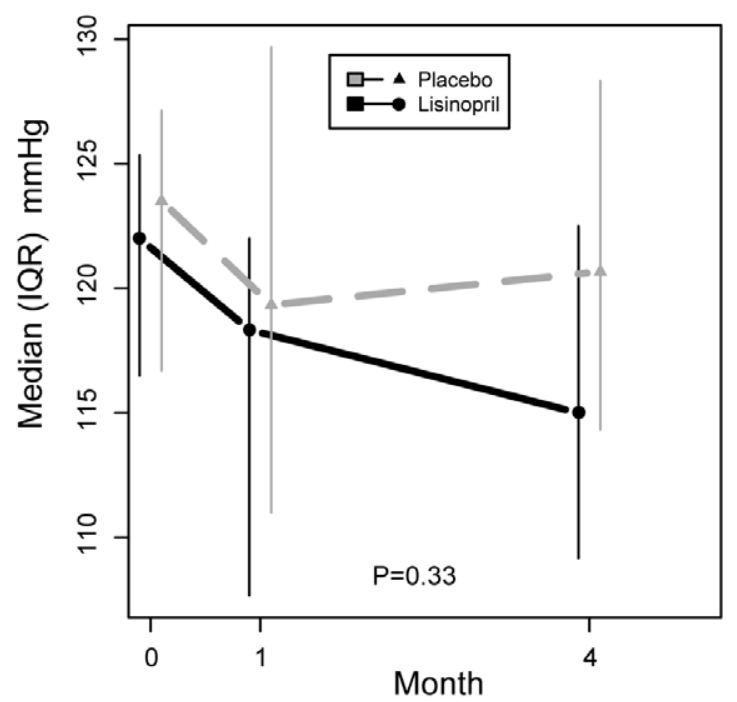

C) hsCRP

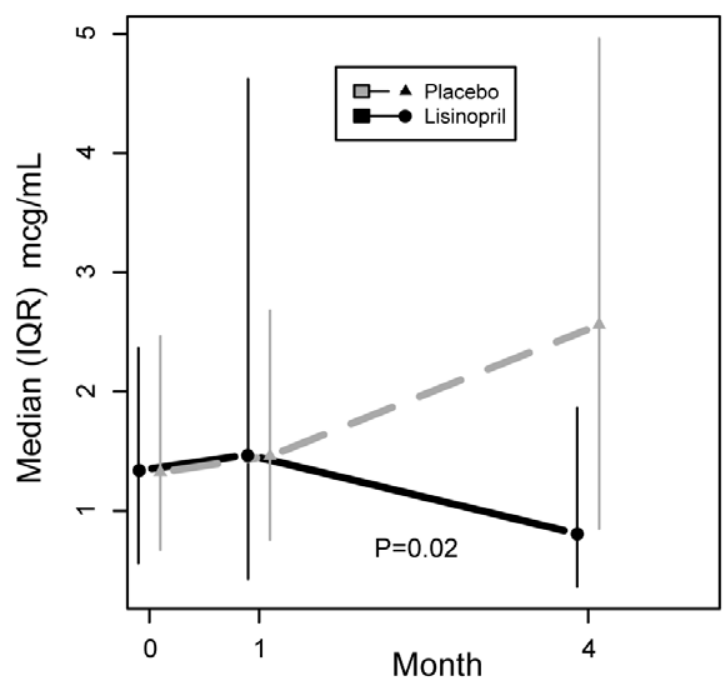

E) TNF-a

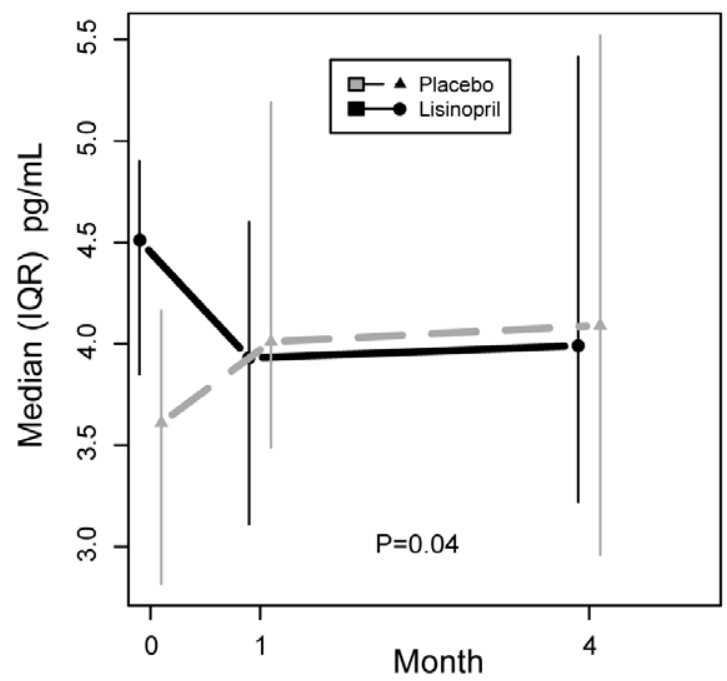

B) Diastolic Blood Pressure

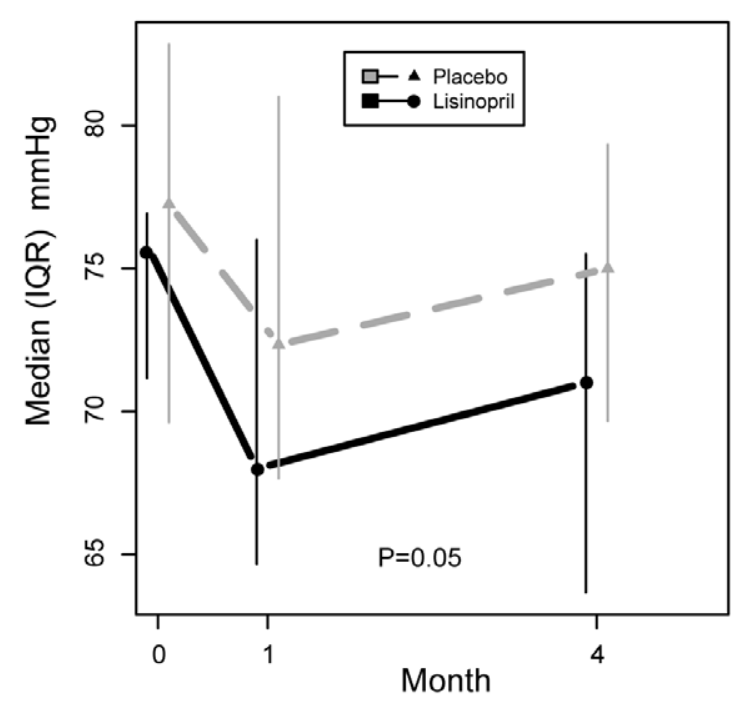

D) IL-6

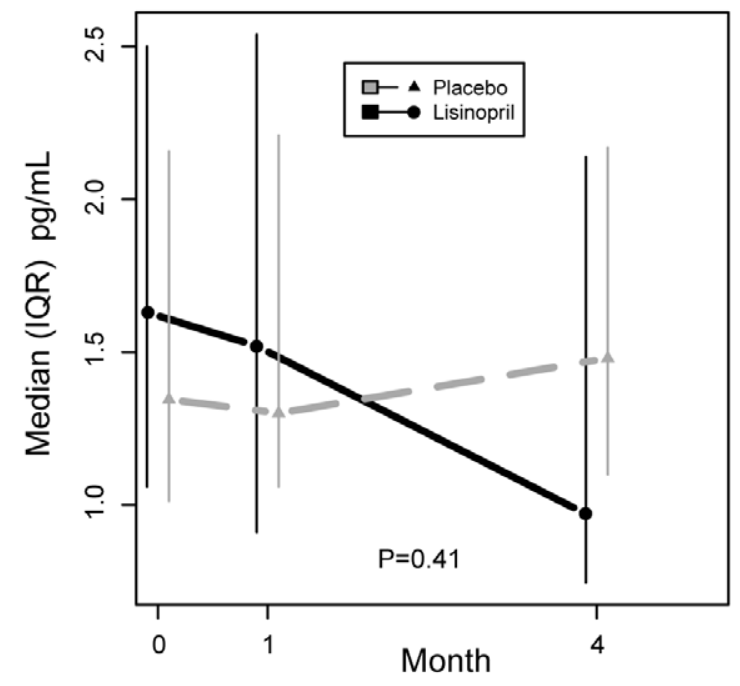

F) Inflammatory Rank Score

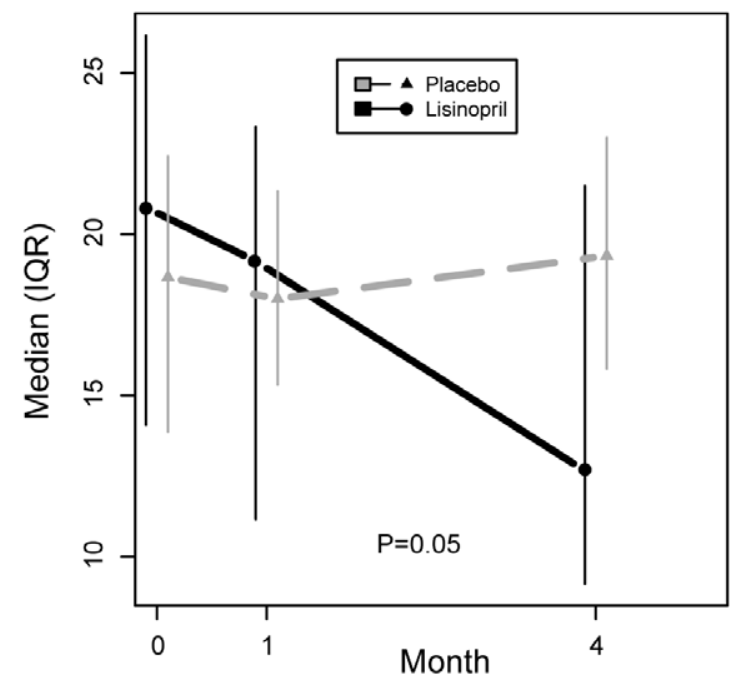

October 2012 | Volume 7 | Issue 10 | e46894 
Figure 2. Median (IQR) Levels of Blood Pressure and Biomarkers of Inflammation for Lisinopril versus L-placebo Treatment Groups. Values are plotted at baseline and month 1 and 4 for: a) systolic blood pressure, b) diastolic blood pressure, c) hsCRP level, d) IL- 6 level, e) TNF- $\alpha$ level, and f) the inflammatory rank score (the rank sum for hsCRP, IL-6 and TNF- $\alpha$ levels). Lisinopril group is in black (solid line) and L-placebo in grey (dashed line). P-values represent treatment comparisons from longitudinal models that estimate the average differences between groups over follow-up after adjusting for baseline value (see text for absolute estimates). doi:10.1371/journal.pone.0046894.g002

\section{Treatment Effect of Pravastatin on Cholesterol}

There were no significant differences in total cholesterol, LDLC, triglycerides, HDL-C, or total-to-HDL-C ratio between pravastatin and P-placebo groups across follow-up visits (Table 4).

\section{Inflammatory Biomarkers}

Baseline levels of hsCRP, IL-6 and TNF- $\alpha$ are reported (table 1), and there were no differences between treatment groups at study entry. At baseline, 13 (35\%) participants had hsCRP levels categorized as low-risk for CVD $(<1.0 \mathrm{mg} / \mathrm{L})$, with the remaining either average- $(\mathrm{n}=18$, hsCRP $1.0-3.0 \mathrm{mg} / \mathrm{L})$ or high- $(\mathrm{n}=6$, hsCRP $>3.0 \mathrm{mg} / \mathrm{L}$ ) GVD risk [19]. Figure 2 presents the median values during follow-up. The average change (decrease) from baseline during follow-up (estimated from longitudinal models) was greater for lisinopril compared to L-placebo for hsCRP $(-0.54 \mu \mathrm{g} / \mathrm{mL}, \quad 95 \% \quad$ CI $\quad-0.90, \quad-0.32 ; \mathrm{p}=0.02), \quad$ IL-6 $(-0.88 \mathrm{pg} / \mathrm{mL}, \quad 95 \% \quad$ CI $-1.21, \quad 0.64 ; \quad \mathrm{p}=0.41), \quad \mathrm{TNF}-\alpha$ $(-0.84 \mathrm{pg} / \mathrm{mL}, 95 \%$ CI $-0.99,-0.71 ; \mathrm{p}=0.04)$. These differences correspond to relative reductions for the lisinopril group of $42 \%$ for hsCRP and $23 \%$ for TNF- $\alpha$. The treatment effect for hsCRP was not apparent until month $4(-0.34 \mu \mathrm{g} / \mathrm{mL} ; \mathrm{p}=0.02)$, whereas the decline in TNF- $\alpha$ was present by month $1(-0.81 \mathrm{pg} /$ $\mathrm{mL} ; \mathrm{p}=0.02)$. Finally, the inflammatory score improved over follow-up for the group randomized to lisinopril vs. L-placebo $(-2.81,95 \%$ CI -5.68 to $0.05 ; \mathrm{p}=0.054)$.

There was no evidence of a pravastatin treatment effect on any of the inflammatory biomarkers at month 1 , month 4 , or averaged over both follow-up visits. There were also no differences in the lisinopril treatment effect on biomarker changes between those receiving or not receiving pravastatin (data not shown).

\section{Discussion}

This feasibility study of lisinopril and/or pravastatin as a CVD prevention strategy for HIV positive persons taking effective ART with viral suppression provides important information for future trials directed at BP and lipid changes among HIV positive patients who do not have an indication for these drugs. We found that adherence to lisinopril (at $10 \mathrm{mg}$ daily) was less than for matched L-placebo, and as a consequence blood pressure lowering was modest. Improvements in blood lipids were not evident with pravastatin (at $20 \mathrm{mg}$ daily) though this effect may have been limited by the relatively low potency at this dose as well as the small sample size. Importantly, among these virologically suppressed patients, we also found that lisinopril led to short-term improvements in biomarkers of systemic inflammation (hsCRP and TNF- $\alpha$ ).

Epidemiologic data suggests persons with HIV infection have an approximate 2-fold increased risk for CVD, when compared to the general population [16,20,21]. Whether absolute CVD event rates will continue to differ and widen further over time is unclear, given some evidence that more aggressive management of traditional risk factors in contemporary HIV care has attenuated CVD risk [22]. Newer antiretroviral medications may also continue to reduce metabolic complications. However, even with optimal management of $\mathrm{BP}$ and cholesterol to levels below clinical treatment thresholds based on risk, factors unique to HIV disease still appear to result in excess CVD events $[4,23,24]$. Furthermore, by focusing on extreme elevations in individual risk factors there are missed opportunities to reduce CVD risk through modest simultaneous reductions in multiple risk factors. This, combined with the observation that CVD risk can be reduced among persons without clinically overt CVD by lowering BP within normotensive values or lowering LDL-C beyond $130 \mathrm{mg} / \mathrm{dL}$ [25,26,27,28], motivates the strategy of combining low-doses of various GVD prevention medications into a singly daily pill as prevention (i.e., the polypill) $[9,10,29]$. Our data provide some support that such a study is feasible in individuals with HIV infection, but, consistent with data from general population [30,31] issues of tolerability, adherence, and potency will need to be carefully considered. Future studies like this should consider a run-in period to assess adherence or use of better-tolerated medications with similar mechanisms (e.g., angiotensin receptor blockers).

The proportion of ART-treated HIV infected patients in clinical practice that currently have no indication for treatment with an ACE-I or a statin likely varies widely by setting. The reported prevalence for dyslipidemia (e.g., cholesterol $>200-240 \mathrm{mg} / \mathrm{dL}$, HDL $<35 \mathrm{mg} / \mathrm{dL}$, receipt of lipid-lowering therapy or clinical diagnosis) has ranged from 30-35\% [16,22,23,24]. For hypertension ( $\mathrm{BP} \geq 140 / 90 \mathrm{mmHg}$, receiving BP lowering therapy or a clinical diagnosis), it is between $10-30 \%$, and for a prior history of CVD between 5-10\% [16,22,23,24]. When one also excludes persons at very low risk CVD risk (e.g., unlikely to benefit from aggressive prevention efforts), the target population for a preemptive GVD prevention strategy will likely include much less than half of patients in most HIV clinical settings. Furthermore, CVD prevention treatments will likely be most effectively implemented if they target patients receiving ART whose risk for AIDS complications is low. Despite these potential exclusions, the difference between a low-burden of GVD risk factors and

Table 3. The Treatment Effect of Lisinopril $(n=17)$ Versus L-Placebo $(n=17)$ on Blood Pressure.

\begin{tabular}{|c|c|c|c|c|}
\hline & Month 1 Change ${ }^{1}(95 \% \mathrm{Cl})$ & Month 4 Change $^{1}(95 \% \mathrm{Cl})$ & $\begin{array}{l}\text { Average Change During } \\
\text { Follow-up }(95 \% \mathrm{CI})^{2}\end{array}$ & $p$-value ${ }^{2}$ \\
\hline Systolic BP (mmHg) & $-3.4(-8.9,2.1)$ & $-1.8(-9.4,5.8)$ & $-2.63(-8.1,2.8)$ & 0.33 \\
\hline Diastolic BP (mmHg) & $-3.3(-6.9,0.2)$ & $-3.3(-7.3,0.7)$ & $-3.3(-6.5,-0.1)$ & 0.05 \\
\hline
\end{tabular}


Table 4. The Treatment Effect of Pravastatin $(n=18)$ Versus P-Placebo $(n=16)$ on Blood Lipid Levels $(n=34)$.

\begin{tabular}{|c|c|c|c|c|}
\hline & Month 1 Change ${ }^{1}(95 \% \mathrm{Cl})$ & $\begin{array}{l}\text { Month } 4 \text { Change }{ }^{1} \\
(95 \% \mathrm{CI})\end{array}$ & $\begin{array}{l}\text { Average Change During } \\
\text { Follow-up }(95 \% \mathrm{CI})^{2}\end{array}$ & $p$-value ${ }^{2}$ \\
\hline Total Chol. (mg/dL) & $0.08(-12.42,12.59)$ & $-1.75(-18.74,15.25)$ & $9.5(-2.5,21.5)$ & 0.96 \\
\hline LDL-C (mg/dL) & $-2.29(-16,79,12.22)$ & $-0.62(-13.22,11.98)$ & $5.5(-6.2,17.2)$ & 0.87 \\
\hline HDL-C (mg/dL) & $5.74(-1.75,13.24)$ & $0.97(-8.42,10.36)$ & $3.81(-2.3,10.0)$ & 0.28 \\
\hline TC/HDL-C & $-0.42(-0.81,-0.04)$ & $-0.07(-1.11,0.97)$ & $0.01(-0.6,0.6)$ & 0.39 \\
\hline
\end{tabular}

1) Regression models of treatment difference, adjusted for baseline level.

$\left.{ }^{2}\right)$ Longitudinal mixed models of changes over both follow-up time points, adjusted for baseline level.

doi:10.1371/journal.pone.0046894.t004

optimally managed risk factors still has substantial implications for longer-term CVD risk over a lifetime [32]. Defining the appropriate target population that optimizes the net benefit-risk balance will be an important goal for future HIV-related CVD prevention studies.

Inflammation is a key factor in the pathogenesis of cardiovascular disease and a hallmark of HIV infection that persists despite effective treatment with ART for years [2,5]. The reasons for chronic immune activation and inflammation are multi-factorial, but potential drivers include residual low-level HIV replication, translocation of microbial products across damaged mucosal barriers, the presence of co-pathogens (e.g., herpes viruses or hepatitis B or $\mathrm{C}$ ), as well as metabolic complications (e.g., increased visceral adiposity) $[33,34,35,36]$. In this context, antiinflammatory treatments are particularly attractive candidates for HIV-related CVD prevention, whether or not they target HIVspecific mechanisms or down-regulate inflammatory pathways more broadly. ACE-I and statins have been associated with antiinflammatory effects $[13,14,15]$. We found that among persons with HIV infection, lisinopril use was associated with a decline in biomarkers of systemic inflammation. Favorable changes were evident in spite of suboptimal adherence. High-sensitivity CRP, specifically, is elevated with HIV infection and associated with risk for CVD among both HIV-infected and uninfected persons $[2,3,20]$.

Our findings were limited by the small sample size. Confidence intervals are wide and we may have missed important treatment effects. Low power also limited our ability to detect treatment interactions. The lack of a treatment effect of pravastatin may be due to the low potency of this statin, as we did not detect changes in cholesterol or lipoproteins. Given the approach we were studying (i.e., adding pravastatin as primary prevention to asymptomatic patients) we chose a starting dose (e.g., $20 \mathrm{mg}$ ) to minimize risk/tolerability and our short-term follow-up duration

\section{References}

1. Grinspoon SK, Grunfeld C, Kotler DP, Currier JS, Lundgren JD, et al. (2008) State of the science conference: Initiative to decrease cardiovascular risk and increase quality of care for patients living with HIV/AIDS: executive summary. Circulation 118: 198-210.

2. Neuhaus J, Jacobs DR Jr, Baker JV, Calmy A, Duprez D, et al. (2010) Markers of inflammation, coagulation, and renal function are elevated in adults with HIV infection. J Infect Dis 201: 1788-1795.

3. Kuller LH, Tracy R, Belloso W, De Wit S, Drummond F, et al. (2008) Inflammatory and coagulation biomarkers and mortality in patients with HIV infection. PLoS Med 5: e203.

4. Kaplan RC, Sinclair E, Landay AL, Lurain N, Sharrett AR, et al. (2011) T Cell Activation and Senescence Predict Subclinical Carotid Artery Disease in HIVInfected Women. J Infect Dis 203: 452-463.

5. Hunt PW, Brenchley J, Sinclair E, McCune JM, Roland M, et al. (2008) Relationship between $\mathrm{T}$ cell activation and CD4+ $\mathrm{T}$ cell count in HIVseropositive individuals with undetectable plasma HIV RNA levels in the absence of therapy. J Infect Dis 197: 126-133. precluded dose escalation. Other HIV studies using higher doses (i.e., pravastatin $40 \mathrm{mg}$ daily) or other statins (e.g., atorvastatin and rosuvastatin) have demonstrated reductions in measures of immune activation or inflammation [13,37].

In summary, our results support the feasibility of conducting further studies of similar adjunct treatments that may have multiple beneficial effects such as reducing BP and systemic inflammation among HIV positive patients. Adherence concerns with lisinopril in this context suggest other, more tolerable medications with similar effects on the renin-angiotensin-aldosterone-system (e.g., angiotensin receptor blockers), may be a more effective strategy. Ultimately, in addition to larger feasibility studies, HIV clinical outcome trials will have to performed to assess the risk/benefit of such adjunctive treatment strategies.

\section{Supporting Information}

\section{Checklist S1 GONSORT Ghecklist.}

(DOC)

\section{Protocol S1 Trial Protocol.}

(PDF)

\section{Acknowledgments}

The authors would like to thank all study participants, and our research staff: Bette Bordenave, Edie Gunderson, Rachel Givot, Deb Woods, Mark Olson, Miki Olson, Chelsea Robinson, Jack Hall and Candace Nelson.

\section{Author Contributions}

Conceived and designed the experiments: JB KHH DD RT RG KH JN. Performed the experiments: JB RP FR RT. Analyzed the data: JB KHH JN. Contributed reagents/materials/analysis tools: RT. Wrote the paper: JB KHH RP DD KH JN.

6. Stein JH, Hadigan CM, Brown TT, Chadwick E, Feinberg J, et al. (2008) Prevention strategies for cardiovascular disease in HIV-infected patients. Circulation 118: e54-60.

7. Lewington S, Clarke R, Qizilbash N, Peto R, Collins R (2002) Age-specific relevance of usual blood pressure to vascular mortality: a meta-analysis of individual data for one million adults in 61 prospective studies. Lancet 360: 1903-1913.

8. Ormiston J, Webster M (2007) Absorbable coronary stents. Lancet 369: 1839 1840.

9. Jackson R, Lawes CM, Bennett DA, Milne RJ, Rodgers A (2005) Treatment with drugs to lower blood pressure and blood cholesterol based on an individual's absolute cardiovascular risk. Lancet 365: 434-441.

10. Law MG (2006) Cardiovascular complications of HIV: an overview of risk and a novel approach to prevention - the HIV polypill study. Curr Opin HIV AIDS 1: 482-487.

11. Baigent C, Keech A, Kearney PM, Blackwell L, Buck G, et al. (2005) Efficacy and safety of cholesterol-lowering treatment: prospective meta-analysis of data 
from 90,056 participants in 14 randomised trials of statins. Lancet 366: 12671278.

12. Law MR, Morris JK, Wald NJ (2009) Use of blood pressure lowering drugs in the prevention of cardiovascular disease: meta-analysis of 147 randomised trials in the context of expectations from prospective epidemiological studies. BMJ 338: b1665.

13. Ganesan A, Crum-Cianflone N, Higgins J, Qin J, Rehm C, et al. (2011) High dose atorvastatin decreases cellular markers of immune activation without affecting HIV-1 RNA levels: results of a double-blind randomized placebo controlled clinical trial. The Journal of infectious diseases 203: 756-764.

14. Jain MK, Ridker PM (2005) Anti-inflammatory effects of statins: clinical evidence and basic mechanisms. Nature reviews Drug discovery 4: 977-987.

15. Montecucco F, Pende A, Mach F (2009) The renin-angiotensin system modulates inflammatory processes in atherosclerosis: evidence from basic research and clinical studies. Mediators of inflammation 2009: 752406.

16. Freiberg M, McGinnis K, Butt A, Goetz M, Brown S, et al. (2011) HIV in associated with clinically confirmed myocardial infarction after adjustment for smoking and other risk factors. 18th Conference on Retroviruses and Opportunistic Infections. Boston, MA, USA.

17. Anderson KM, Wilson PW, Odell PM, Kannel WB (1991) An updated coronary risk profile. A statement for health professionals. Circulation 83: 356-362.

18. Kaplan RC, Landay AL, Hodis HN, Gange SJ, Norris PJ, et al. (2012) Potential cardiovascular disease risk markers among HIV-infected women initiating antiretroviral treatment. Journal of acquired immune deficiency syndromes.

19. Pearson TA, Mensah GA, Alexander RW, Anderson JL, Cannon RO 3rd, et al (2003) Markers of inflammation and cardiovascular disease: application to clinical and public health practice: A statement for healthcare professionals from the Centers for Disease Control and Prevention and the American Heart Association. Circulation 107: 499-511.

20. Triant VA, Meigs JB, Grinspoon SK (2009) Association of C-reactive protein and HIV infection with acute myocardial infarction. J Acquir Immune Defic Syndr 51: 268-273.

21. Obel N, Thomsen HF, Kronborg G, Larsen CS, Hildebrandt PR, et al. (2007) Ischemic heart disease in HIV-infected and HIV-uninfected individuals: a population-based cohort study. Clin Infect Dis 44: 1625-1631.

22. Sabin CA, d'Arminio Monforte A, Friis-Moller N, Weber R, El-Sadr WM, et al. (2008) Changes over time in risk factors for cardiovascular disease and use of lipid-lowering drugs in HIV-infected individuals and impact on myocardial infarction. Clin Infect Dis 46: 1101-1110.

23. Triant VA, Regan S, Lee H, Sax PE, Meigs JB, et al. (2010) Association of Immunologic and Virologic Factors With Myocardial Infarction Rates in a US Healthcare System. J Acquir Immune Defic Syndr.

24. Baker JV, Henry WK, Patel P, Bush TJ, Conley LJ, et al. (2011) Progression of carotid intima-media thickness in a contemporary human immunodeficiency virus cohort. Clinical infectious diseases : an official publication of the Infectious Diseases Society of America 53: 826-835.

25. Anderson KM, Odell PM, Wilson PW, Kannel WB (1991) Cardiovascular disease risk profiles. American heart journal 121: 293-298.

26. Neaton JD, Blackburn H, Jacobs D, Kuller L, Lee DJ, et al. (1992) Serum cholesterol level and mortality findings for men screened in the Multiple Risk Factor Intervention Trial. Multiple Risk Factor Intervention Trial Research Group. Arch Intern Med 152: 1490-1500.

27. Cruickshank J (2001) The lowering of blood pressure after stroke. Lancet 358: 1994; author reply 1994-1995.

28. Heart Protection Study Collaborative Group. (2002) MRC/BHF Heart Protection Study of cholesterol lowering with simvastatin in 20,536 high-risk individuals: a randomised placebo-controlled trial. Lancet 360: 7-22.

29. Lonn E, Bosch J, Teo KK, Pais P, Xavier D, et al. (2010) The polypill in the prevention of cardiovascular diseases: key concepts, current status, challenges, and future directions. Circulation 122: 2078-2088.

30. Yusuf S, Pais P, Afzal R, Xavier D, Teo K, et al. (2009) Effects of a polypill (Polycap) on risk factors in middle-aged individuals without cardiovascular disease (TIPS): a phase II, double-blind, randomised trial. Lancet 373: 13411351

31. Rodgers A, Patel A, Berwanger O, Bots M, Grimm R, et al. (2011) An international randomised placebo-controlled trial of a four-component combination pill ("polypill") in people with raised cardiovascular risk. PLoS One 6: e19857.

32. Berry JD, Dyer A, Cai X, Garside DB, Ning H, et al. (2012) Lifetime risks of cardiovascular disease. The New England journal of medicine 366: 321-329.

33. Naeger DM, Martin JN, Sinclair E, Hunt PW, Bangsberg DR, et al. (2010) Cytomegalovirus-specific $\mathrm{T}$ cells persist at very high levels during long-term antiretroviral treatment of HIV disease. PLoS One 5: e8886.

34. Brenchley JM, Price DA, Schacker TW, Asher TE, Silvestri G, et al. (2006) Microbial translocation is a cause of systemic immune activation in chronic HIV infection. Nat Med 12: 1365-1371.

35. Boger MS, Shintani A, Redhage LA, Mitchell V, Haas DW, et al. (2009) Highly sensitive C-reactive protein, body mass index, and serum lipids in HIV-infected persons receiving antiretroviral therapy: a longitudinal study. J Acquir Immune Defic Syndr 52: 480-487.

36. Buzon MJ, Massanella M, Llibre JM, Esteve A, Dahl V, et al. (2010) HIV-1 replication and immune dynamics are affected by raltegravir intensification of HAART-suppressed subjects. Nature Medicine 16: 460-465.

37. Neogi U, Shet A, Shamsundar R, Ekstrand ML (2011) Selection of nonnucleoside reverse transcriptase inhibitor-associated mutations in HIV-1 subtype C: evidence of etravirine cross-resistance. AIDS 25: 1123-1126. 